NUS/HEP/94201

hep-th/9407142

\title{
Generalized $q$-Oscillators and their Hopf Structures
}

\author{
C.H. Oh ${ }^{\dagger}$ and K. Singh $\ddagger$ \\ Department of Physics \\ Faculty of Science \\ National University of Singapore \\ Lower Kent Ridge, Singapore 0511 \\ Republic of Singapore
}

\begin{abstract}
We study the relationships among the various forms of the $q$ oscillator algebra and consider the conditions under which it supports a Hopf structure. We also present a generalization of this algebra together with its corresponding Hopf structure. Its multimode extensions are also considered.
\end{abstract}

To appear in J. Phys.A: Math. Gen.

†E-mail: PHYOHCH@NUSVM.NUS.SG

†E-mail: PHYSINGH@NUSVM.NUS.SG 


\section{Introduction}

Quantum groups or more precisely the quantized universal enveloping algebras $\mathcal{U}_{q}(\mathcal{L})$ of Lie algebras $\mathcal{L}$ first emerged as the basic algebraic structures in the study of the quantum Yang-Baxter equations [1]. It was later shown by Drinfeld [2] that these structures could be described by a general class of associative algebras, called Hopf algebras, which are neither commutative nor cocommutative. Essentially the non-cocommutativity is achieved by introducing a free parameter $q$ which is usually called the deformation parameter.

Now one of the most well studied example is that of the quantum group $\mathcal{U}_{q}(\operatorname{su}(2)$ ) (or sometimes denoted as $\left.\mathrm{su}_{q}(2)\right)$ which was first considered by Skylanin [3] and independently by Kulish and Reshetikhin [4]. Recently this algebra has been realized in terms of a $q$ analogue of the bosonic creation and annhiliation operators [5,6]. Indeed, Macfarlane [5] introduced these $q$-oscillators $a, a^{+}$by considering their action on a Hilbert space with basis $\{\mid n>\}, n=0,1,2, \ldots$ defined by

$$
a|0>=0, \quad| n>=([n] !)^{-1 / 2}\left(a^{+}\right)^{n} \mid 0>,
$$

where

$$
[n]=\frac{q^{n}-q^{-n}}{q-q^{-1}} \quad \text { and } \quad[n] !=[n][n-1][n-2] \ldots[1]
$$

Then by setting

$$
\begin{aligned}
& a^{+} a=[N], \\
& a a^{+}=[N+1],
\end{aligned}
$$

where $N$ satisfies

$$
N|n>=n| n>
$$

he was able to furnish a representation for the $q$-oscillators:

$$
\begin{aligned}
a^{+} \mid n> & =[n+1]^{1 / 2} \mid n+1>, \\
a \mid n> & =[n]^{1 / 2} \mid n-1>.
\end{aligned}
$$

Moreover in this representation, one also has the following relations:

$$
\begin{aligned}
& a a^{+}-q a^{+} a=q^{-N}, \\
& a a^{+}-q^{-1} a^{+} a=q^{N},
\end{aligned}
$$

besides

$$
\left[N, a^{+}\right]=a^{+}, \quad[N, a]=-a .
$$


Biedenharn [6] also independently arrived at similar results but instead of starting with relations (2), he postulated (4) and (6) with $q$ replaced by $q^{1 / 2}$.

By using the Jordan-Schwinger construction, they gave a bosonic realization of $\operatorname{su}_{q}(2)$. Conversely, the $q$-oscillators can also be obtained directly from the usual representation of $\mathrm{su}_{q}(2) . \mathrm{Ng}$ [7] showed that by setting $j \rightarrow \infty, m \rightarrow \infty$, in the basis vectors spanning the Hilbert space of $\mathrm{su}_{q}(2)$, the $q$-oscillators can be obtained which satisfy relations (2).

Although the $q$-oscillators have been primarily used in giving realizations of quantum groups, it itself may support a quantum group structure. Indeed, Hong Yan [8] showed that the $q$-oscillator algebra when expressed in a symmetric form could be endowed with a noncocommutative Hopf structure. Instead of relations (2), he considered the the commutator $\dagger$

$$
\left[a, a^{+}\right]=[N+1]-[N] .
$$

Then together with relations (6) he was able to construct a non-trivial Hopf algebra. It is worth noting that while relations (2) imply relation (7), the converse is not true; it is only in the representation (3) that the two are equivalent. In fact the same holds for relations (4) and (5) in regard to (2) or (7).

In the following section, we discuss some issues pertaining to this inequivalence. In particular, we study the relationships among the various forms of the $q$-oscillators. Moreover, we also clarify some misleading notions in the literature about the $q$-oscillator algebra when regarded as a quantum group. In section 3 we present a generalized deformed oscillator algebra which also has a Hopf structure. Here, Hong Yan's algebra is recoverd as a special case. The representation of this generalized algebra is also furnished. In section 4 we consider its multimode extensions. Besides a set consisting of mutually commuting oscillators, we also present a multidimensional quantum group based on our generalization.

\section{2. q-Oscillator Algebras}

The $q$-oscillator algebra consists of three elements $a, a^{+}$and $N$ defined by (6) together with one of the relations (2), (4), (5), (7). In the following we will examine how the various forms namely, (2), (4), (5), and (7) of the oscillator algebra are related to each other. Here relations (6) will be implicitly assumed as part of the algebra. For clarity, we consider two at a time.

$\dagger$ In ref.[8] the right hand side is expressed as $\left[N+\frac{1}{2}\right]-\left[N-\frac{1}{2}\right]$. Here we have made the replacement $N \rightarrow N+\frac{1}{2}$, to be consistent with the notation that we have adopted in this paper. 
Case (i): Between (2) and (4).

Starting with (2), we show that it implies (4). Indeed, by substituting (2) into the LHS of (4), we have

$$
a a^{+}-q a^{+} a=[N+1]-q[N]=q^{-N} .
$$

which is precisely the RHS of (4). On the other hand to see whether (4) implies (2) we construct the Casmir operator for the algebra defined by (4) and (6). Now, it is easy to verify that

$$
\mathcal{C}_{(4)}=q^{-N}\left([N]-a^{+} a\right)
$$

commutes with all the operators i.e. $a, a^{+}, N$. Thus one can write

$$
a^{+} a=[N]-q^{N} \mathcal{C}_{(4)}
$$

Moreover, we also have, using (4)

$$
a a^{+}=q a^{+} a+q^{-N}=[N+1]-q^{N+1} \mathcal{C}_{(4)} .
$$

It is apparent then that (4) implies (2) only if $\mathcal{C}_{(4)}=0$. To show inequivalence it is sufficient to show that there exist representations of $(4)$ in which $\mathcal{C}_{(4)}$ is non zero. To this end, we have for $n=0,1,2 \ldots$ (see ref. [9])

$$
\begin{aligned}
a^{+} \mid n> & =q^{-\nu_{0} / 2}[n+1]^{1 / 2} \mid n+1>, \\
a \mid n> & =q^{-\nu_{0} / 2}[n]^{1 / 2} \mid n-1>, \\
N \mid n> & =\left(\nu_{0}+n\right) \mid n>
\end{aligned}
$$

which suitably represents (4) and (6). Note that this representation carries a free parameter $\nu_{0}$ and is more general then (3) above. In this representation one has

$$
\mathcal{C}_{(4)}\left|n>=q^{-\nu_{0}}\left[\nu_{0}\right]\right| n>
$$

which shows that for $\nu_{0} \neq 0, \mathcal{C}_{(4)}$ cannot be regarded as the null operator. Thus we can surmise that (4), in general, do not imply (2).

Case (ii): Between (2) and (5).

Using arguments paralleling above, it is easy to show that (2) implies (5) but not the converse. Here $\mathcal{C}_{(5)}$ is similar to $\mathcal{C}_{(4)}$ with $q \leftrightarrow q^{-1}$. 
Case (iii) : Between (2) and (7).

It is obvious that (2) implies (7). For the converse we construct the Casmir operator $\mathcal{C}_{(7)}$ for (7) which reads as

$$
\mathcal{C}_{(7)}=[N]-a^{+} a
$$

or

$$
a^{+} a=[N]-\mathcal{C}_{(7)} .
$$

Using (7), we also have

$$
a a^{+}=[N+1]-\mathcal{C}_{(7)} .
$$

From these it is clear that (7) implies (2) only if $\mathcal{C}_{(7)}=0$. Again for non-equivalence, it suffices to show that $\mathcal{C}_{(7)}$ is not zero in some representation. In this case one can construct the following representation:

$$
\begin{aligned}
a^{+} \mid n> & =\left(\left[n+1-\nu_{0}\right]+\left[\nu_{0}\right]\right)^{1 / 2} \mid n+1>, \\
a \mid n> & =\left(\left[n-\nu_{0}\right]+\left[\nu_{0}\right]\right)^{1 / 2} \mid n-1>, \\
N \mid n> & =\left(n-\nu_{0}\right) \mid n>,
\end{aligned}
$$

for $n=0,1,2 \ldots$ in which

$$
\mathcal{C}_{(7)}\left|n>=-\left[\nu_{0}\right]\right| n>.
$$

It is evident then that $\mathcal{C}_{(7)} \neq 0$ for $\nu_{0} \neq 0$.

Case (iv): Between (4) and (5).

From (9b) and (9c) we have

$$
\begin{aligned}
a a^{+}-q^{-1} a^{+} a & =[N+1]-q^{N+1} \mathcal{C}_{(4)}-q^{-1}\left([N]-q^{N} \mathcal{C}_{(4)}\right) \\
& =q^{N}-\left(q-q^{-1}\right) q^{N} \mathcal{C}_{(4)}
\end{aligned}
$$

which shows that (4) is not equivalent to $(5)$ since $\mathcal{C}_{(4)} \neq 0$ in general. Similarly (5) does not imply (4).

Case (v): Between (4) and (7).

From $(9 \mathrm{~b})$ and $(9 \mathrm{c})$ we obtain

$$
a a^{+}-a^{+} a=[N+1]-[N]-(q-1) q^{N} \mathcal{C}_{(4)}
$$

which means that (4) does not imply (7). Conversely from (12b) and (12c) we have

$$
\begin{aligned}
a a^{+}-q a^{+} a & =[N+1]-q[N]-\mathcal{C}_{(7)}+q \mathcal{C}_{(7)} \\
& =q^{-N}+(q-1) \mathcal{C}_{(7)}
\end{aligned}
$$


which again demonstrates the inequivalence.

Case (vi) : Between (5) and (7).

Arguments and conclusions similar to case (v) with $q \leftrightarrow q^{-1}$.

From the results above we can surmise that although the various forms are interchangeable in the representation (3), they are nevertheless inequivalent at the algebraic level. This distinction becomes particularly important when one deals with pure algebraic constructs. For instance, when we are considering the Hopf structure of the $q$-oscillators, it is necessary to distinguish the relation in question. Now, due to the equivalence of (2), (4), (5) and (7) in the representation (3), it is sometimes implied that they are all Hopf algebras. Indeed, it has been claimed (see ref. [10]) that relations (4) together with (5) which imply (2) and hence (7) have a Hopf structure defined by $\dagger$ :

$$
\begin{aligned}
\Delta\left(a^{+}\right) & =\left(a^{+} \otimes q^{1 / 2(N+1 / 2)}+i q^{-1 / 2(N+1 / 2)} \otimes a^{+}\right) e^{-i \theta / 2}, \\
\Delta(a) & =\left(a \otimes q^{1 / 2(N+1 / 2)}+i q^{-1 / 2(N+1 / 2)} \otimes a\right) e^{-i \theta / 2}, \\
\Delta(N) & =N \otimes \mathbf{1}+\mathbf{1} \otimes N+\gamma \mathbf{1} \otimes \mathbf{1}, \\
\Delta(\mathbf{1}) & =\mathbf{1} \otimes \mathbf{1}, \\
\epsilon\left(a^{+}\right) & =\epsilon(a)=0, \\
\epsilon(N) & =-\gamma, \\
S\left(a^{+}\right) & =-q^{1 / 2} a^{+}, \quad S(a)=-q^{-1 / 2} a, \\
S(N) & =-N-2 \gamma \mathbf{1}, \quad S(\mathbf{1})=\mathbf{1},
\end{aligned}
$$

where $\gamma=\frac{1}{2}-\frac{i \theta}{\ln q}$ and $\theta=(\pi / 2)+2 \pi l, l \in \mathbf{Z}$. Here the maps $\Delta, \epsilon, S$ which are the coproduct, counit and antipode respectively, satisfy

$$
\Delta(a b)=\Delta(a) \Delta(b), \quad \epsilon(a b)=\epsilon(a) \epsilon(b), \quad S(a b)=S(b) S(a)
$$

for any two elements $a, b$ of the Hopf algebra. In other words they are algebra homomorphisms (antihomomorphism for the case of $S$ ). Although these constitute a Hopf structure for relation (7) together with (6), they do not for relations (4), (5), and (2). To see why this is so, consider relation (4) as an example. By applying $\Delta$ on both sides we have using (19),

$$
\begin{aligned}
\Delta(a) \Delta\left(a^{+}\right)-q \Delta\left(a^{+}\right) \Delta(a) & =-i\left\{q^{-N} \otimes q^{N+1 / 2}+i(1-q) q^{-1 / 2} q^{-1 / 2 N} a \otimes q^{1 / 2 N} a^{+}\right. \\
& \left.-q^{-(N+1 / 2)} \otimes q^{-N}+i(1-q) q^{1 / 2} q^{-1 / 2 N} a^{+} \otimes q^{1 / 2 N} a\right\}
\end{aligned}
$$

$\dagger$ In ref. [10] $q^{1 / 2}$ instead of $q$ is used. 
whereas

$$
\Delta\left(q^{-N}\right)=i q^{-1 / 2} q^{-N} \otimes q^{-N}
$$

which means that

$$
\Delta(a) \Delta\left(a^{+}\right)-q \Delta\left(a^{+}\right) \Delta(a) \neq \Delta\left(q^{-N}\right) .
$$

Similarly one can easily verify that $\Delta$ is also incompatible with (2) and (5). Thus the Hopf structure is valid only for relation (7) and not for the rest.

\section{Generalized $q$-Oscillator Algebra}

Recently some authors [11] have considered a generalized version of (4),

$$
a a^{+}-q a^{+} a=q^{\alpha N+\beta}
$$

together with (6) and attempted to give it a Hopf structure. However, the coproduct defined there fails the compatibility requirement, in the same way as (18a) and (18b) failed for relations (4), (5) and (2). Specifically, the proposed coproduct,

$$
\begin{aligned}
\Delta\left(a^{+}\right) & =a^{+} \otimes q^{1 / 2(\alpha N+\beta)}+q^{-1 / 2(\alpha N+\beta)} \otimes a^{+}, \\
\Delta(a) & =a \otimes q^{1 / 2(\alpha N+\beta)}+q^{-1 / 2(\alpha N+\beta)} \otimes a, \\
\Delta(N) & =N \otimes \mathbf{1}+\mathbf{1} \otimes N+(\beta / \alpha) \mathbf{1} \otimes \mathbf{1},
\end{aligned}
$$

fails with respect to $(23)$ :

$$
\Delta(a) \Delta\left(a^{+}\right)-q \Delta\left(a^{+}\right) \Delta(a) \neq \Delta\left(q^{\alpha N+\beta}\right) .
$$

In this section we furnish a generalized version of the $q$-oscillator algebra which can be endowed with a Hopf structure.

As seen from the previous section, among the various forms of the $q$-oscillator algebra relation (7) is the only version that supports a Hopf structure. So it is conceivable that any generalization would most likely be based on (7) rather than (4). To write down a generalized version of (7), it is instructive to consider again the relationship between (4) and $(7)$. Instead of using a representation in which $\mathcal{C}_{(4)}$ is zero (see (16)), one can also obtain (7) by considering both (4) and (5). When taken together they imply (2) which in turn imply (7). It is worth noting that (5) is the $q \leftrightarrow q^{-1}$ analogue of (4). Now let us apply this procedure to (23). Since $\alpha$ and $\beta$ are arbitrary we can replace $\alpha \rightarrow-\alpha$ and $\beta \rightarrow-\beta$ and rewrite $(23)$ as

$$
a a^{+}-q a^{+} a=q^{-\alpha N-\beta}
$$


Its $q \leftrightarrow q^{-1}$ analogue is given by

$$
a a^{+}-q^{-1} a^{+} a=q^{\alpha N+\beta}
$$

which together with (26) imply that

$$
\begin{aligned}
& a a^{+}=[\alpha N+\beta], \\
& a^{+} a=[\alpha N+\beta+1] .
\end{aligned}
$$

This in turn leads us to

$$
\left[a, a^{+}\right]=[\alpha N+\beta+1]-[\alpha N+\beta]
$$

or

$$
\left[a, a^{+}\right]=\left[\alpha N+\beta_{1}\right]-\left[\alpha N+\beta_{2}\right]
$$

where

$$
\beta_{1}-\beta_{2}=1
$$

which is a generalization of (7) we sought. To be as general as possible we will also modify (6) somewhat by introducing a free parameter $\eta$ into the commutation relations:

$$
\left[N, a^{+}\right]=\eta a^{+}, \quad[N, a]=-\eta a
$$

It is worth noting that the algebra composed of (30) and (31) admits a non-trivial central term which is given by

$$
\mathcal{C}=a^{+} a-\frac{1}{2} \frac{\sinh (\epsilon(\alpha N+\beta+1 / 2-\alpha \eta / 2))}{\cosh (\epsilon / 2) \sinh (\epsilon \alpha \eta / 2)} .
$$

Now it is also important to note that, as in the case between (2) and (7), relations (30) do not necessarily imply (28) although the latter have been used in constructing the former. This can be demonstrated easily by constructing a representation of (30) and (31) in which (26), (27) and (28) do not hold individually. Indeed, in the basis $\{\mid n>\}$, $n=0,1,2, \ldots$, a representation of $(30)$ and $(31)$ is given by

$$
\begin{aligned}
a \mid n> & =\left\{\frac{\cosh \left(\epsilon \alpha\left(\nu_{0}+(n-1) \eta / 2\right)+\epsilon(\beta+1 / 2)\right) \sinh (\epsilon \eta \alpha n / 2)}{\cosh (\epsilon / 2) \sinh (\epsilon \eta \alpha / 2)}\right\}^{1 / 2} \mid n-1>, \\
a^{+} \mid n> & =\left\{\frac{\cosh \left(\epsilon \alpha\left(\nu_{0}+n \eta / 2\right)+\epsilon(\beta+1 / 2)\right) \sinh (\epsilon \eta \alpha(n+1) / 2)}{\cosh (\epsilon / 2) \sinh (\epsilon \eta \alpha / 2)}\right\}^{1 / 2} \mid n+1>, \\
N \mid n> & =\left(\nu_{0}+n \eta\right) \mid n>,
\end{aligned}
$$


where we have taken $\epsilon=\ln q$ and $\nu_{0}$ is a free parameter which characterizes the representation. In this representation, it not difficult to see the inequivalence between (30) and any one among (26)-(28). This is true even when $\eta$ is set to 1 .

Now let us turn to the Hopf structure associated with (30) and (31). We start by considering the associative algebra $\mathcal{H}$ generated by $\left\{\mathbf{1}, a^{+}, a, N\right\}$ and postulating the following for the coproduct, counit and antipode:

$$
\begin{aligned}
\Delta\left(a^{+}\right) & =c_{1} a^{+} \otimes q^{\alpha_{1} N}+c_{2} q^{\alpha_{2} N} \otimes a^{+}, \\
\Delta(a) & =c_{3} a \otimes q^{\alpha_{3} N}+c_{4} q^{\alpha_{4} N} \otimes a, \\
\Delta(N) & =c_{5} N \otimes \mathbf{1}+c_{6} \mathbf{1} \otimes N+\gamma \mathbf{1} \otimes \mathbf{1}, \\
\Delta(\mathbf{1}) & =\mathbf{1} \otimes \mathbf{1}, \\
\epsilon\left(a^{+}\right) & =c_{7} \\
\epsilon(N) & =c_{9}, \quad \epsilon(a)=c_{8}, \\
S\left(a^{+}\right) & =-c_{10} a^{+}, \quad S(a)=-c_{11} a, \\
S(N) & =-c_{12} N+c_{13} \mathbf{1}, \quad S(\mathbf{1})=\mathbf{1} .
\end{aligned}
$$

Here $c_{i}, i=1,2, \ldots 13, \alpha_{i}, i=1,2, \ldots 4$ and $\gamma$ are constants to be determined. These constants are obtained by requiring that $\Delta, \epsilon$ and $S$ satisfy the coassociativity, counit and antipode axioms respectively:

$$
\begin{aligned}
& (\mathrm{id} \otimes \Delta) \Delta(h)=(\Delta \otimes \mathrm{id}) \Delta(h), \\
& (\mathrm{id} \otimes \epsilon) \Delta(h)=(\epsilon \otimes \mathrm{id}) \Delta(h)=h, \\
& m(\mathrm{id} \otimes S) \Delta(h)=m(S \otimes \mathrm{id}) \Delta(h)=\epsilon(h) \mathbf{1},
\end{aligned}
$$

where $h \in \mathcal{H}$ and $m: \mathcal{H} \otimes \mathcal{H} \rightarrow \mathcal{H}$ is the multiplication map. By substituting the different generators of $\mathcal{H}$ into (35) and noting that

$$
\begin{aligned}
a^{+} q^{\alpha N} & =q^{-\alpha \eta} q^{\alpha N} a^{+} \\
a q^{\alpha N} & =q^{\alpha \eta} q^{\alpha N} a
\end{aligned}
$$

for an arbitrary $\alpha$, we obtain

$$
\begin{aligned}
& c_{1}=q^{\alpha_{1} \gamma}, \quad c_{2}=q^{\alpha_{2} \gamma}, \quad c_{3}=q^{\alpha_{3} \gamma}, \quad c_{4}=q^{\alpha_{4} \gamma} \quad c_{5}=1, \\
& c_{6}=1, \quad c_{7}=0, \quad c_{8}=0, \quad c_{9}=-\gamma, \quad c_{10}=q^{\alpha_{1} \eta}, \\
& c_{11}=q^{-\alpha_{3} \eta}, \quad c_{12}=1, \quad c_{13}=-2 \gamma, \quad \alpha_{2}=-\alpha_{1}, \quad \alpha_{4}=-\alpha_{3},
\end{aligned}
$$


which essentially fixes 15 of the 18 constants in (34). We must also require that $\Delta, \epsilon$ and $S$ be algebra homomorphisms. Here further constraints arise when we set

$$
\Delta(a) \Delta\left(a^{+}\right)-\Delta\left(a^{+}\right) \Delta(a)=\Delta\left(\left[\alpha N+\beta_{1}\right]-\left[\alpha N+\beta_{2}\right]\right)
$$

For this to be satisfied we must impose the following:

$$
\begin{aligned}
& q^{\left(\alpha_{1}-\alpha_{3}\right) \eta}=1, \\
& \alpha_{1}+\alpha_{3}=\alpha, \\
& q^{2 \alpha \gamma}=-q^{\beta_{1}+\beta_{2}} .
\end{aligned}
$$

With these, the homomorphisms $\epsilon$ and $S$ entail no further constraints. For real $q$, eqns. (39) imply that

$$
\alpha_{1}=\alpha_{3}=\frac{1}{2} \alpha
$$

and

$$
\gamma=\frac{\beta_{1}+\beta_{2}}{2 \alpha}-\frac{i(2 k+1) \pi}{2 \alpha \ln q} \quad k \in \mathbf{Z}
$$

which now fixes all the constants in (34).

To summarize briefly, the Hopf structure for $\mathcal{H}$ with defining relations (30) and (31) reads as

$$
\begin{aligned}
\Delta\left(a^{+}\right) & =a^{+} \otimes q^{\frac{1}{2} \alpha(N+\gamma)}+q^{-\frac{1}{2} \alpha(N+\gamma)} \otimes a^{+}, \\
\Delta(a) & =a \otimes q^{\frac{1}{2} \alpha(N+\gamma)}+q^{-\frac{1}{2} \alpha(N+\gamma)} \otimes a, \\
\Delta(N) & =N \otimes \mathbf{1}+\mathbf{1} \otimes N+\gamma \mathbf{1} \otimes \mathbf{1} \\
\Delta(\mathbf{1}) & =\mathbf{1} \otimes \mathbf{1}, \\
\epsilon\left(a^{+}\right) & =\epsilon(a)=0, \quad \epsilon(\mathbf{1})=1 \\
\epsilon(N) & =-\gamma, \\
S\left(a^{+}\right) & =-q^{\frac{1}{2} \alpha \eta} a^{+}, \quad S(a)=-q^{-\frac{1}{2} \alpha \eta} a \\
S(N) & =-N-2 \gamma \mathbf{1}, \quad S(\mathbf{1})=\mathbf{1},
\end{aligned}
$$

where $\gamma$ satisfies (40b). Note that by setting $\eta=1, \beta_{1}=1, \beta_{2}=0, \alpha=1$ and putting $k=2 l, \quad l \in \mathbf{Z}$ in (40b) we recover the Hopf structure associated with (6) and (7).

\section{Multimode $q$-Oscillators}

Various multimode extensions of the $q$-oscillators have been proposed $[4,5,12,13]$. In particular the extension of (4) and (5) or equivalently that of (2) consist of taking $p$ 
independent oscillators (mutually commuting) $\left\{a_{i}, a_{i}^{+}, N_{i} \mid i=1,2, \ldots p\right\}$ with the relations $[13]$

$$
\begin{aligned}
& a_{i} a_{j}^{+}-\left(1+\delta_{i j}(q-1)\right) a_{j}^{+} a_{i}=\delta_{i j} q^{-N_{i}}, \\
& a_{i} a_{j}^{+}-\left(1+\delta_{i j}\left(q^{-1}-1\right)\right) a_{j}^{+} a_{i}=\delta_{i j} q^{N_{i}}, \\
& {\left[a_{i}, a_{j}\right]=\left[a_{i}^{+}, a_{j}^{+}\right]=0,} \\
& {\left[N_{i}, a_{j}\right]=-\delta_{i j} a_{j}, \quad\left[N_{i}, a_{j}^{+}\right]=\delta_{i j} a_{j}^{+} .}
\end{aligned}
$$

Here we present a multimode extension of (30) and show that it also supports a noncocommutative Hopf structure. To this end we propose the following relations for the set of $p$ oscillators:

$$
\begin{aligned}
& {\left[a_{i}, a_{j}^{+}\right]=\left(\left[\alpha_{i} N_{i}+\beta_{i}+1\right]-\left[\alpha_{i} N_{i}+\beta_{i}\right]\right) \delta_{i j},} \\
& {\left[a_{i}, a_{j}\right]=\left[a_{i}^{+}, a_{j}^{+}\right]=0,} \\
& {\left[N_{i}, a_{j}\right]=-\eta_{i} a_{j} \delta_{i j}, \quad\left[N_{i}, a_{j}^{+}\right]=\eta_{i} a_{j}^{+} \delta_{i j}}
\end{aligned}
$$

where $\alpha_{i}, \beta_{i}$ and $\eta_{i}(i=1,2, \ldots p)$ are free parameters. Then it can easily be shown that the associative algebra generated by $\left\{\mathbf{1}, a_{i}, a_{i}^{+}, N_{i}\right\}, i=1,2, \ldots p$ with the above defining relations admits the following non-cocommutative Hopf structure:

$$
\begin{aligned}
\Delta\left(a_{i}^{+}\right) & =a_{i}^{+} \otimes q^{\frac{1}{2} \alpha_{i}\left(N_{i}+\gamma_{i}\right)}+q^{-\frac{1}{2} \alpha_{i}\left(N_{i}+\gamma_{i}\right)} \otimes a_{i}^{+}, \\
\Delta\left(a_{i}\right) & =a_{i} \otimes q^{\frac{1}{2} \alpha_{i}\left(N_{i}+\gamma_{i}\right)}+q^{-\frac{1}{2} \alpha_{i}\left(N_{i}+\gamma_{i}\right)} \otimes a_{i}, \\
\Delta\left(N_{i}\right) & =N_{i} \otimes \mathbf{1}+\mathbf{1} \otimes N_{i}+\gamma_{i} \mathbf{1} \otimes \mathbf{1}, \\
\Delta(\mathbf{1}) & =\mathbf{1} \otimes \mathbf{1}, \\
\epsilon\left(a_{i}^{+}\right) & =\epsilon\left(a_{i}\right)=0, \\
\epsilon\left(N_{i}\right) & =-\gamma_{i}, \\
S\left(a_{i}^{+}\right) & =-q^{\frac{1}{2} \alpha_{i} \eta_{i}} a_{i}^{+}, \quad S(\mathbf{1})=1, \\
S\left(N_{i}\right) & =-N_{i}-2 \gamma_{i} \mathbf{1}, \quad S(\mathbf{1})=-q^{-\frac{1}{2} \alpha_{i} \eta_{i}} a_{i},
\end{aligned}
$$

with

$$
\gamma_{i}=\frac{2 \beta_{i}+1}{2 \alpha_{i}}-\frac{i\left(2 k_{i}+1\right) \pi}{2 \alpha_{i} \ln q} \quad k_{i} \in \mathbf{Z}
$$

In verifying the homomorphism property we have used

$$
\begin{aligned}
a_{i}^{+} q^{\rho N_{j}} & =q^{-\rho \eta_{j} \delta_{i j}} q^{\rho N_{j}} a_{i}^{+}, \\
a_{i} q^{\rho N_{j}} & =q^{\rho \eta_{j} \delta_{i j}} q^{\rho N_{j}} a_{i},
\end{aligned}
$$


for an arbitrary $\rho$. Here the Hopf structure is essentially the Hopf structure of each oscillator taken independently. It is interesting to note that the oscillators can also be coupled in a non-trivial way. To see how this can be accomplished, let us examine relations (39) closely. If we allow $\eta$ to be complex and relate it to $q$ via

$$
\eta=\frac{2 \pi i}{\ln q}
$$

then relation (39a) implies that $\alpha_{1}-\alpha_{3}=l,(l \in \mathbf{Z})$. Now this means that we can assign integer values to $\alpha_{1}$ and $\alpha_{3}$ which in turn allows the indexing of oscillators. For instance, if we set $\alpha_{1}=m$ and $\alpha_{3}=n$ then the oscillators can be indexed as $a_{m}$ and $a_{n}^{+}$respectively. This effectively permits a number of oscillators to be considered together. Moreover, with $\alpha$ also being integer valued, as a consequence of (39b), the commutation relations between the various oscillators become non trivial. As for $(39 \mathrm{c})$, we have

$$
\beta_{1}+\beta_{2}=2\left(\alpha_{1}+\alpha_{3}\right) \gamma+i \frac{(2 k+1) \pi}{\ln q} \quad k \in \mathbf{Z}
$$

Then by putting $k=0$ (for simplicity) and using (30b), we obtain

$$
\begin{aligned}
& \beta_{1}=\left(\alpha_{1}+\alpha_{3}\right) \gamma+\frac{i \pi}{2 \ln q}+\frac{1}{2}, \\
& \beta_{2}=\left(\alpha_{1}+\alpha_{3}\right) \gamma+\frac{i \pi}{2 \ln q}-\frac{1}{2} .
\end{aligned}
$$

With these, we have $\dagger$

$$
\begin{aligned}
{\left[a_{m}, a_{n}^{+}\right] } & =\left[(m+n)(N+\gamma)+\frac{i \pi}{2 \ln q}+\frac{1}{2}\right]-\left[(m+n)(N+\gamma)+\frac{i \pi}{2 \ln q}-\frac{1}{2}\right] \\
& =i \frac{\sinh (\epsilon(m+n)(N+\gamma))}{\cosh (\epsilon / 2)}
\end{aligned}
$$

where we have taken $q=e^{\epsilon}$. Thus the commutation relations for a system of $p$ oscillators can be written as

$$
\begin{aligned}
& {\left[a_{m}, a_{n}^{+}\right]=i \frac{\sinh (\epsilon(m+n)(N+\gamma))}{\cosh (\epsilon / 2)}} \\
& {\left[a_{m}, a_{n}\right]=\left[a_{m}^{+}, a_{n}^{+}\right]=0,} \\
& {\left[N, a_{m}\right]=-\frac{2 \pi i}{\ln q} a_{m}, \quad\left[N, a_{m}^{+}\right]=\frac{2 \pi i}{\ln q} a_{m}^{+}}
\end{aligned}
$$

$\dagger$ Here we have set $\alpha_{1}=m$ and $\alpha_{3}=n$ and the corresponding oscillators by $a_{m}$ and $a_{n}^{+}$ respectively. 
where $m, n=1,2, \ldots p$. It is important to note that unlike the previous case we have only one $N$ operator. The corresponding Hopf structure is then given by

$$
\begin{array}{rlrl}
\Delta\left(a_{m}^{+}\right) & =a_{m}^{+} \otimes q^{m(N+\gamma)}+q^{-m(N+\gamma)} \otimes a_{m}^{+}, \\
\Delta\left(a_{m}\right) & =a_{m} \otimes q^{m(N+\gamma)}+q^{-m(N+\gamma)} \otimes a_{m}, \\
\Delta(N) & =N \otimes \mathbf{1}+\mathbf{1} \otimes N+\gamma \mathbf{1} \otimes \mathbf{1}, \\
\Delta(\mathbf{1}) & =\mathbf{1} \otimes \mathbf{1}, \\
\epsilon\left(a_{m}^{+}\right) & =\epsilon\left(a_{m}\right)=0, \\
\epsilon(N) & =-\gamma, & & \\
S\left(a_{m}^{+}\right) & =-a_{m}^{+}, & & S\left(a_{m}\right)=-a_{m}, \\
S(N) & =-N-2 \gamma \mathbf{1}, & S(\mathbf{1})=\mathbf{1} .
\end{array}
$$

\section{Conclusion}

In this paper we have considered the various forms of the $q$-oscillator algebra and shown that, contrary to the commonly held notion, they are actually not equivalent. It is also pointed out that the Hopf structure found for one of these versions does not extend to the rest by virtue of this inequivalence. For the algebra that is a quantum group, we have given its generalization together with the associated Hopf structure. Based on this generalization we have also furnished two multimode extensions. In the first example, we have considered a set of (mutually commuting) independent oscillators and shown that the Hopf structure of each oscillator system extends naturally to the multimode case. For the second example, we have presented a Hopf algebra comprising of a set of non-commuting oscillators. 


\section{REFERENCES}

[1.] Faddev L D 1982 Les Houches Lectures (Elsevier,Amsterdam, 1984)

Kulish P P and Sklyanin E K 1982 Lecture Notes in Physics Vol.151 (Springer, Berlin);

[2.] Drinfeld V G 1986 Proc. Intern. Congress of Mathematicians (Berkley) Vol. 1 pg 798 ;

[3.] Sklyanin E K 1982, Funct. Anal. Appl. 16 262;

[4.] Kulish P P and Reshetikhin N Y (1983) J. Sov. Math. 23 2435;

[5.] Macfarlane A J 1989 J. Phys. A22 4581;

[6.] Biedenharn L C 1989 J. Phys. A22 L873;

[7.] Ng Y J 1990 J. Phys. A23 1023;

[8.] Hong Yan 1990 J. Phys. A23 L1155;

[9.] Rideau G 1992 Lett. Math. Phys. 24 147;

[10.] Floreanini R and Vinet L 1991 Lett. Math. Phys. 22 45;

[11.] Chung W, Chung K, Nam S and Um C 1993 Phys. Lett. A 183 363;

[12.] Pusz W and Woronowicz S L (1989) Rep. Math. Phys. 27 231;

[13.] Hayashi T (1990) Commun. Math. Phys. 127 129; 